Article

\title{
Different Apoptotic Pathways Activated by Oxaliplatin in Primary Astrocytes vs. Colo-Rectal Cancer Cells
}

\section{Matteo Zanardelli ${ }^{1}$, Laura Micheli ${ }^{1}$, Raffaella Nicolai ${ }^{2}$, Paola Failli ${ }^{1}$, Carla Ghelardini ${ }^{1}$ and Lorenzo Di Cesare Mannelli ${ }^{1, *}$}

1 Department of Neuroscience, Psychology, Drug Research and Child Health - Neurofarba Pharmacology and Toxicology Section, University of Florence, Florence 50139, Italy; E-Mails: matteo.zanardelli@unifi.it (M.Z.); laura.micheli@unifi.it (L.M.); paola.failli@unifi.it (P.F.); carla.ghelardini@unifi.it (C.G.)

2 Sigma-Tau Industrie Farmaceutiche Riunite S.p.A., Via Pontina km 30,400, I-00040 Pomezia, Rome 00040, Italy; E-Mail: Raffaella.Nicolai@sigma-tau.it

* Author to whom correspondence should be addressed; E-Mail: lorenzo.mannelli@unifi.it; Tel.: +39-055-4271-316; Fax: +39-055-4271-280.

Academic Editor: Ritva Tikkanen

Received: 22 January 2015 / Accepted: 2 March 2015 / Published: 9 March 2015

\begin{abstract}
Oxaliplatin-based chemotherapy improves the outcomes of metastatic colorectal cancer patients. Its most significant and dose-limiting side effect is the development of a neuropathic syndrome. The mechanism of the neurotoxicity is unclear. The limited knowledge about differences existing between neurotoxic and antitumor effects hinders the discovery of effective and safe adjuvant therapies. In vitro, we suggested cell-specific activation apoptotic pathways in normal nervous cells (astrocytes) vs. colon-cancer cells (HT-29). In the present research we compared the apoptotic signals evoked by oxaliplatin in astrocytes and HT-29 analyzing the intrinsic and extrinsic apoptotic pathways. In astrocytes, oxaliplatin induced a mitochondrial derangement measured as cytosolic release of cytochrome $\mathrm{C}$, increase in superoxide anion levels and decreased expression of the antiapoptotic protein Bcl-2. Caspase-8, a main initiator of the extrinsic process remained unaltered. On the contrary, in HT-29 oxaliplatin increased caspase- 8 activity and Bid expression, thus activating the extrinsic apoptosis, while the Bcl-2 increased expression blocked the mitochondrial damage. Data suggest the preferred activation of the intrinsic apoptosis as oxaliplatin damage signaling in normal nervous cells. The extrinsic pathway prevails in tumor cells indicating a possible strategy for planning new molecules to treat oxaliplatin-dependent neurotoxicity without negatively influence chemotherapy.
\end{abstract}


Keywords: glia; HT-29; neuropathic pain; intrinsic apoptotic pathway; extrinsic apoptotic pathway; oxaliplatin

\section{Introduction}

Oxaliplatin was successfully introduced for the management of advanced colorectal cancer, the second leading cause of cancer death in Western countries [1,2]. This antineoplastic agent differs from previous platinum compounds for the configuration of the amino substituents $[3,4]$ and, characteristically, its major dose-limiting side effect is neurotoxicity that leads to the development of peripheral neuropathy $[5,6]$. The poor knowledge about the mechanisms of oxaliplatin neurotoxicity limits the development of effective adjuvant therapies thus making chemotherapy-induced neuropathies an unmet medical need. Moreover, the pharmacological approach is complicated by the fundamental necessity to not interfere with the antitumoral effects.

Recently, we highlighted the redox unbalance as a target for the management of oxaliplatin neurotoxicity, and the natural antioxidant compound silibinin was suggested to prevent nervous damage and pain in a rat model of oxaliplatin neuropathy [7,8]. In a cellular model of oxaliplatin-neurotoxicity (primary astrocyte cell culture), silibinin, as well as $\alpha$-tocopherol, exerted cytoprotective properties reducing the oxidative damage and limiting the activation of caspase- 3 , the downstream effector of apoptotic processes [9]. Interestingly, antioxidants were unable to reduce caspase- 3 activation induced by oxaliplatin in the human adenocarcinoma colorectal cancer cell line HT-29 [9]. The different effect observed in astrocytes and in HT-29 lead us to hypothesize that oxaliplatin may evoke distinct apoptotic signals in normal vs. tumoral cells. On the other hand, the intrinsic and the extrinsic apoptotic pathways mediated by a mitochondrial derangement and by death receptors, respectively, have as common effector caspase 3 [10,11].

Aimed to individuate new and specific biological targets for the treatment of oxaliplatin neurotoxicity, specific markers of the two apoptotic pathways (extensively reviewed in [12]) were studied in primary cultured astrocytes in comparison with HT-29 cells. In particular, the mitochondrial dysfunction was studied by measuring the release of cytochrome $\mathrm{C}$ from mitochondria to the cytosol, the superoxide anion $\left(\mathrm{O}_{2}{ }^{-}\right)$levels [13-15] and the expression of the antiapoptotic protein Bcl-2 [16]. Moreover, the protein expression levels were evaluated for the initiator of the extrinsic apoptotic process death receptor 5 (DR5) [17,18] and Bid, pro-apoptotic protein activated by caspase- 8 and able to transfer the apoptotic information to the intrinsic process [19]. Finally, the activation of caspase-8, central hallmark of the extrinsic pathway was measured $[11,20]$.

\section{Results}

Aimed to evaluate the regulation of the apoptotic processes mediated by oxaliplatin, specific effectors of the intrinsic and extrinsic apoptotic pathways were measured in primary rat astrocytes in comparison to HT-29 cells. Oxaliplatin concentration was chosen on the basis of previous published data [9]. Moreover, the comparison of astrocyte and HT-29 cell viability, after $24 \mathrm{~h}$ incubation with increasing concentrations of oxaliplatin, revealed a similar response in the different cell types 
(Supplementary Material, Table S1). The treatment with oxaliplatin $100 \mu \mathrm{M}$ for $8 \mathrm{~h}$ did not alter cell viability, whereas is allows observing increased caspase-3 activity in astrocytes as well as in HT-29. The pro-apoptotic effect of oxaliplatin was comparable in both cell types [9].

In astrocytes, $8 \mathrm{~h}$ incubation with $100 \mu \mathrm{M}$ oxaliplatin, affected mitochondrial functionality. The immunolabeling of cytochrome $\mathrm{C}$ displayed a punctuate staining in control condition that evolved in a diffuse cytosolic pattern after oxaliplatin treatment (Figure 1).

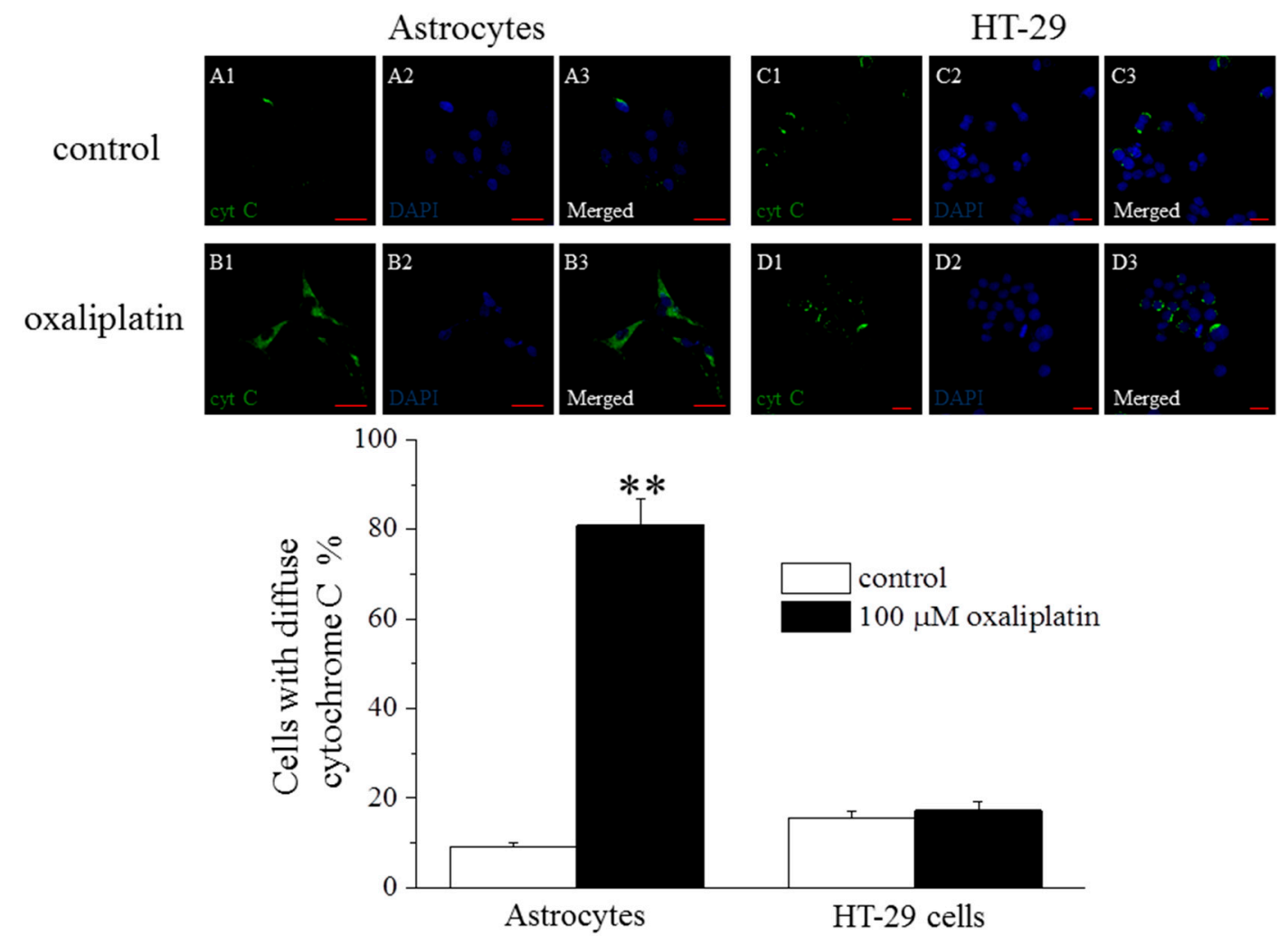

Figure 1. Cytosolic release of cytochrome C. Astrocytes $\left(5 \times 10^{4}\right.$ cells/slide $)$ and HT-29 $\left(5 \times 10^{4}\right.$ cells/slide) were exposed to $100 \mu \mathrm{M}$ oxaliplatin for $8 \mathrm{~h}$. Specimens were stained with anti-cytochrome $\mathrm{C}$ and a secondary antibody conjugated with Alexa Fluor 488 (green) and DAPI (blue) for nucleus visualization. (A1-A3) control astrocytes; (B1-B3) oxaliplatin-treated astrocytes; (C1-C3) control HT-29; (D1-D3) oxaliplatin-treated HT-29. Calibration: $20 \mu \mathrm{m}$. Bars represent the mean \pm S.E.M of cells displaying a diffuse cytosolic distribution of cytochrome $\mathrm{C}$ as percentage of total analyzed cells. Cells were counted using the "cell counter" plugin of ImageJ 1.33, free-share image analysis software (ImageJ, NIH, Bethesda, MD, USA). At least three fields (40X 0.75NA objective) per slide and two slides for each condition were analyzed, repeating the experiment three times. ${ }^{* *} p<0.01 v s$. control.

The release of cytochrome $\mathrm{C}$ from mitochondria to the cytosol was observed in 197 out of 247 treated cells and in 21 out of 253 control cells. On the contrary, oxaliplatin $(100 \mu \mathrm{M}, 8 \mathrm{~h})$ did not alter cytochrome C localization in HT-29 (Figure 1).

In glial cells the mitochondrial alterations were also highlighted by measuring the redox unbalance. Superoxide anion production $\left(\mathrm{O}_{2}{ }^{-}\right)$was increased by oxaliplatin $(100 \mu \mathrm{M}, 4 \mathrm{~h})$ by about 1.5 times (in comparison to the basal level of control condition, $17.9 \pm 0.3 \mu \mathrm{M} / \mathrm{mg}$ protein/4 h; Figure 2). 
In HT-29 cells, the chemotherapic agent did not induce any increase in superoxide anion level as measured in astrocyte cultures. To note, the $\mathrm{O}_{2}{ }^{-}$basal level in the tumoral cells was significantly higher than those detected in the astrocyte cultures $(37.8 \pm 2.1 \mu \mathrm{M} / \mathrm{mg}$ protein $/ 4 \mathrm{~h}$; Figure 2$)$.

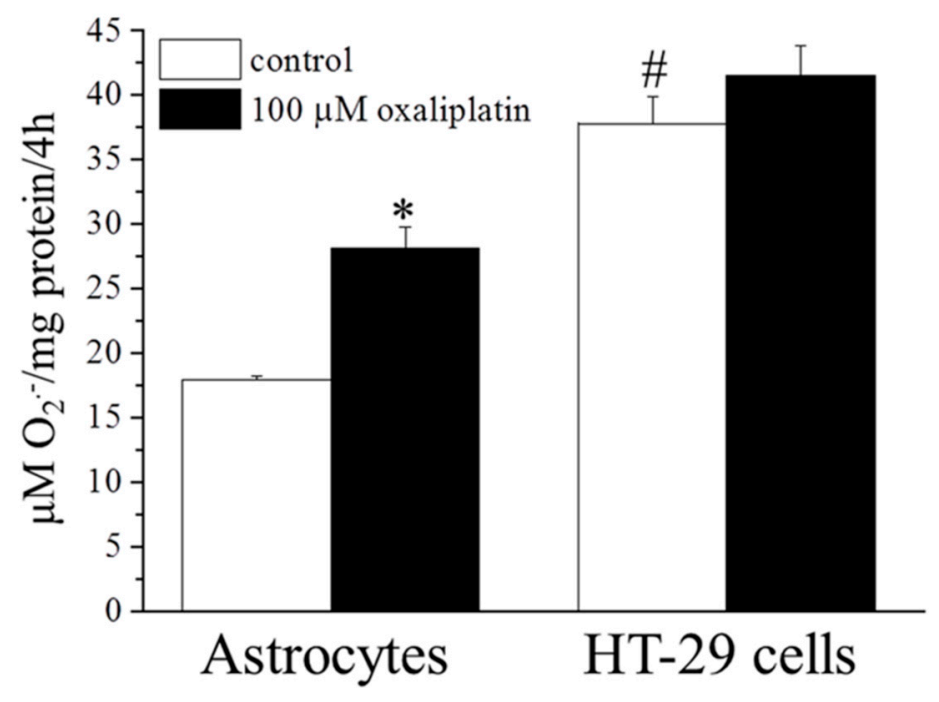

Figure 2. $\mathrm{O}_{2} \cdot{ }^{-}$concentrations. Astrocytes $\left(5 \times 10^{5}\right.$ cells/well $)$ and HT-29 $\left(3 \times 10^{5}\right.$ cells/well $)$ were exposed to $100 \mu \mathrm{M}$ oxaliplatin for $4 \mathrm{~h} . \mathrm{O}_{2}{ }^{-}$concentration was evaluated by cytochrome $\mathrm{C}$ assay. The nonspecific absorbance was measured in the presence of superoxide dismutase (SOD; $300 \mathrm{mU} / \mathrm{mL}$ ) and subtracted from the total value. Values are expressed as $\mu \mathrm{M} / \mathrm{mg}$ protein $/ 4 \mathrm{~h}$. Bars represent the mean \pm S.E.M. of three experiments. ${ }^{*} p<0.05 v s$. control and ${ }^{\#} p<0.05$ vs. astrocytes control.

Evaluating protein expression by Western blot analysis, in basal conditions Bcl-2 was higher in astrocytes as compared to HT-29 (Figure 3). Incubation with oxaliplatin (100 $\mu \mathrm{M}, 8 \mathrm{~h})$ reduced at about $63 \%$ Bcl-2 protein expression in astrocytes, whereas it increased Bcl-2 protein level up to $145.6 \% \pm 12.1 \%$ in HT-29 cells (Figure 3).

The analysis of extrinsic pathway parameters revealed a selective activation in HT-29 cell culture. The incubation ( $8 \mathrm{~h}$ ) with $100 \mu \mathrm{M}$ oxaliplatin did not significantly alter the protein expression of the DR5 receptor neither in astrocytes nor in HT-29 (Figure 4).

We performed experiments aimed to measure caspase- 8 activity. As shown in Figure 5, caspase- 8 activity in astrocyte was $132.3 \pm 8.9$ arbitrary units/mg proteins in control condition and was not changed by oxaliplatin. On the contrary, in HT-29 oxaliplatin increased the enzyme activity up to $292.9 \pm 25.9$ arbitrary units/mg proteins from the control condition of $194.5 \pm 21.4$ arbitrary units/mg proteins. 


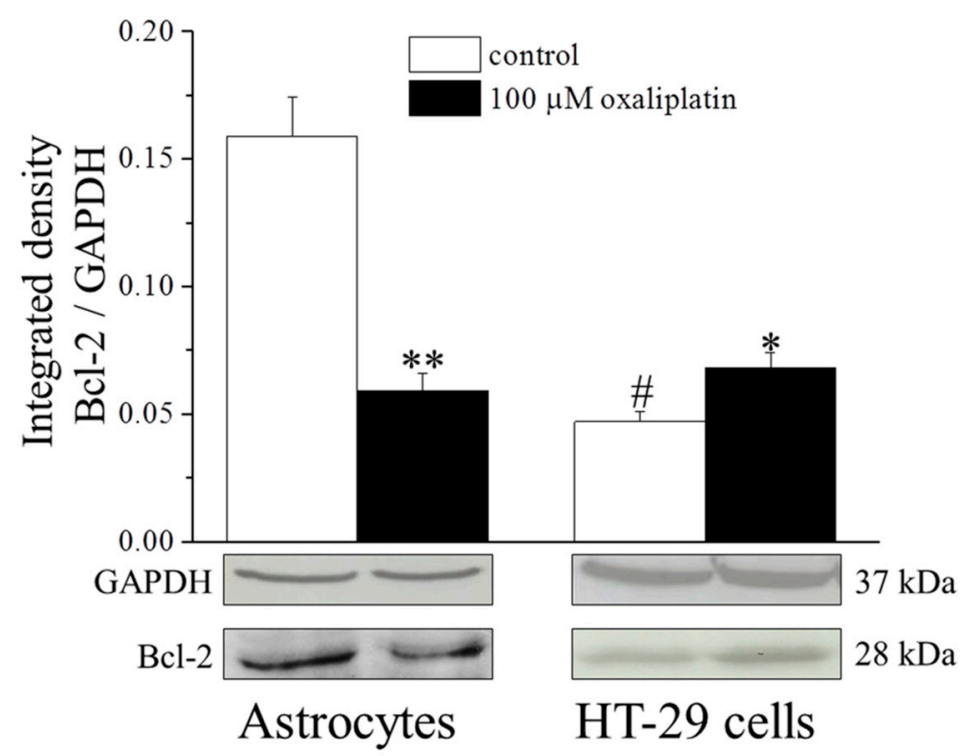

Figure 3. Bcl-2 protein expression. Astrocytes $\left(10^{6}\right.$ cells/flask $)$ and HT-29 $\left(8 \times 10^{5}\right.$ cells/flask $)$ were incubated with $100 \mu \mathrm{M}$ oxaliplatin for $8 \mathrm{~h}$ and then cell lysates were analyzed by Western blot. Densitometric analysis (top) and representative immunoblot (bottom) are shown. Glyceraldehyde-3-Phosphate Dehydrogenase (GAPDH) was used as loading control and GAPDH normalization was performed for each sample. Values are expressed as integrated density making the ratio between Bcl-2 and GAPDH specific band intensities. Bars represent the mean \pm S.E.M. of three experiments. ${ }^{*} p<0.05 v s$. control, ${ }^{* *} p<0.01 v s$. control and ${ }^{\#} p<0.05$ vs. astrocytes control.

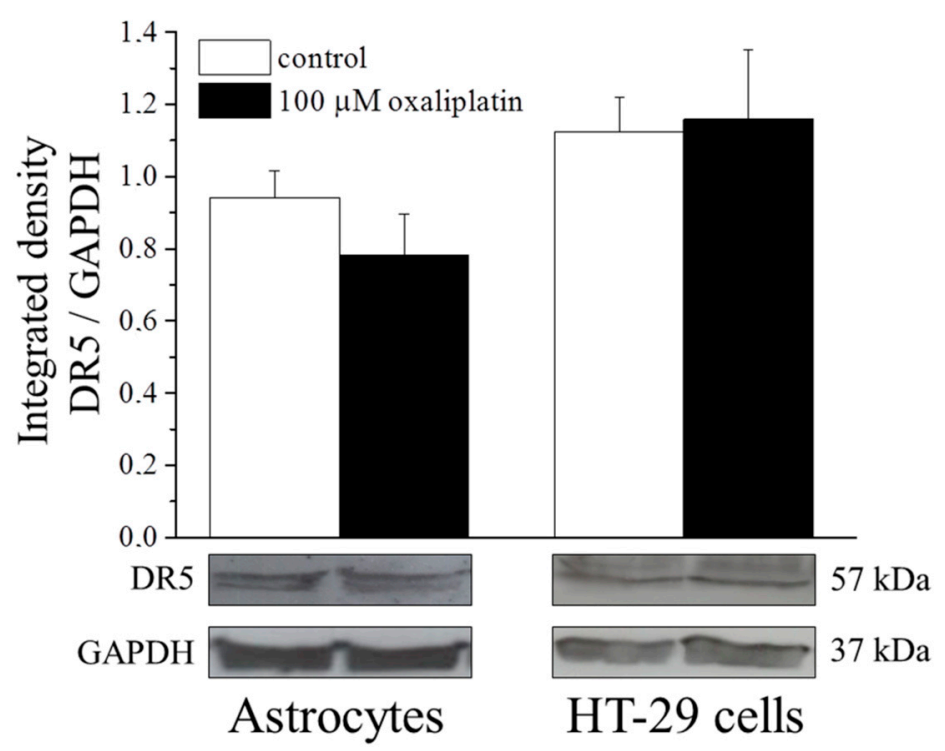

Figure 4. Death receptor 5 (DR5) protein expression. Astrocytes $\left(10^{6}\right.$ cells/flask) and HT-29 $\left(8 \times 10^{5}\right.$ cells/flask) were incubated with $100 \mu \mathrm{M}$ oxaliplatin for $8 \mathrm{~h}$ and then Western blot analysis were performed. Densitometric analysis (top) and representative immunoblot (bottom) are shown. GAPDH was used as loading control and GAPDH normalization was performed for each sample. Values are expressed as integrated density making the ratio between DR5 and GAPDH specific band intensities. Bars represent the mean \pm S.E.M. of three experiments. 
Also oxaliplatin increased caspase- 8 activity in the rat adrenal pheochromocytoma cell line PC12 from the control value of $178.6 \pm 29.6$ arbitrary units $/ \mathrm{mg}$ proteins to $415.8 \pm 28.7$ arbitrary units $/ \mathrm{mg}$ proteins (Figure 5).

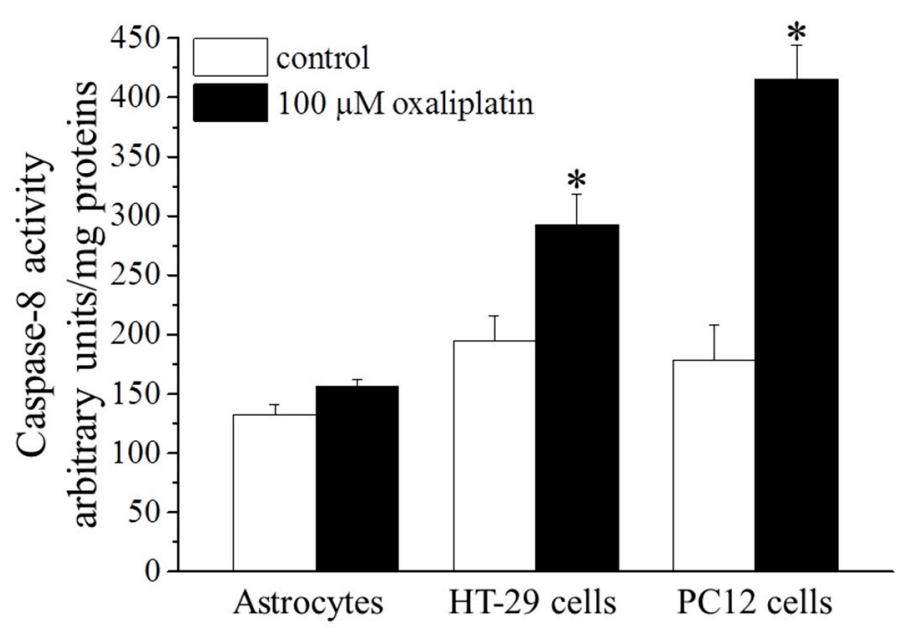

Figure 5. Caspase- 8 activity. Astrocytes $\left(5 \times 10^{5}\right.$ cells/well $)$, HT-29 $\left(3 \times 10^{5}\right.$ cells/well $)$ and PC12 $\left(3 \times 10^{5}\right.$ cells/well $)$ were treated with $100 \mu \mathrm{M}$ oxaliplatin for $8 \mathrm{~h}$. Caspase- 8 activity was expressed as fluorescent arbitrary unit/mg protein. Bars represent the mean \pm S.E.M. of three experiments. ${ }^{*} p<0.05 v s$. control.

Figure 6 shows protein expression levels of Bid evaluated by Western blot. In HT-29, oxaliplatin $(100 \mu \mathrm{M}, 8 \mathrm{~h})$ increased Bid by about $70 \%$. In astrocyte cells, Bid was undetectable in control condition as well as after oxaliplatin treatment.

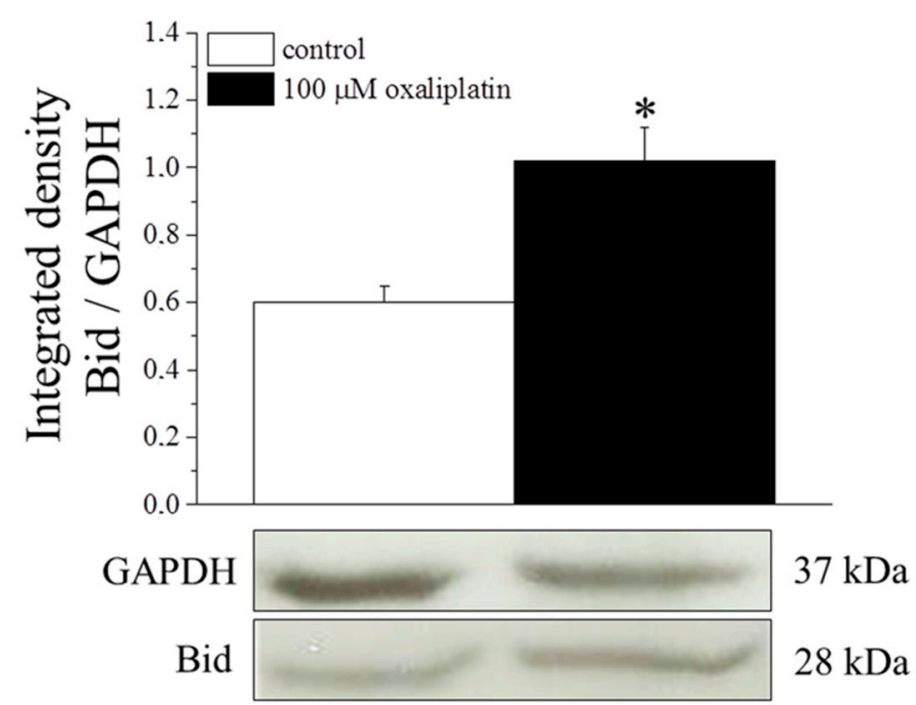

Figure 6. Bid protein expression. HT-29 $\left(8 \times 10^{5}\right.$ cells/flask $)$ were incubated with $100 \mu \mathrm{M}$ oxaliplatin for $8 \mathrm{~h}$ and then cell lysates were analyzed by Western blot. Densitometric analysis (top) and representative immunoblot (bottom) are shown. GAPDH was used as loading control and GAPDH normalization was performed for each sample. Values are expressed as integrated density making the ratio between Bid and GAPDH specific band intensities. Bars represent the mean \pm S.E.M. of three experiments. ${ }^{*} p<0.05$ vs. control. 


\section{Discussion}

Neuropathic syndrome development is one of the main adverse drug reactions that negatively influence the anticancer therapy and drastically reduce the quality of life of the oncologic patients [1,5]. The lack of knowledge about the pathological events at the base of the neurodegenerative phenomenon is primarily responsible for the limited therapeutic resources. The available symptomatic drugs are scarcely effective and the identification of active disease-modifying agents remains the goal of the research in this field. On the other hand, the most important feature of antineuropathic agents candidate to treat cancer patients, is the lack of interaction with chemotherapies maintaining a full effectiveness of the anti-neoplastic potential. A limited knowledge of the distinctions between the biological alterations promoted by the anticancer agents in normal nervous cells and in tumor cells strongly impairs the development of targeted adjuvant therapies.

In the present research, the attention has been focused on oxaliplatin, showing that the apoptosis-inducing mechanism is preferentially mediated in astrocytes by the intrinsic pathway and by the extrinsic in colon cancer cell HT-29.

The intrinsic pathway is promoted by a mitochondrial alteration characterized by membrane potential changes, release of cytochrome $\mathrm{C}$ and redox unbalance [21,22]. In normal glial cells, oxaliplatin promotes the release of cytochrome $\mathrm{C}$ from mitochondria to the cytosol increasing the number of cells showing a diffuse immunoreactivity. Accordingly, Zheng et al. [23] described an oxaliplatin-mediated increase of swollen and vacuolated mitochondria in peripheral nerve axons of neuropathic rats [24]. Mitochondria represent the major source of intracellular ROS, which are by-products of oxidative phosphorylation. ROS excess, or the limited counterbalance by endogenous antioxidant defenses, may damage lipids, proteins, DNA, and mitochondria itself [24]. Oxidative stress can activate the mitochondrial permeability transition pore (mtPTP) creating an open channel across the mitochondrial inner and outer membranes which permits the free diffusion of molecules with a molecular weight lesser than $1500 \mathrm{Da}$. The resulting collapse of trans-membrane electrochemical gradient and the swelling of mitochondria cause the release of cytochrome $C$ [24,25]. Among other ROS, $\mathrm{O}_{2}{ }^{-}$may directly lead to reduced complex I activity, limiting drastically the metabolic role of the organelle [26-28]. In this view, the increased $\mathrm{O}_{2}{ }^{-}$levels registered in nervous cells treated with oxaliplatin [9] and confirmed in the present results, underlines the mitochondrial suffering.

The extent of the intrinsic pathway activation appears marked in astrocytes since levels of the antiapoptotic protein Bcl-2 is reduced after oxaliplatin treatment. Bcl-2 belongs to the Bcl-2 protein superfamily composed by anti-apoptotic and pro-apoptotic factors [26]. Bcl-2 is localized on the external mitochondrial membrane and prevents the release of pro-apoptotic molecules (such as cytochrome C) from the mitochondria to the cytosol [29,30] limiting the voltage dependent anion channel formation and the dysregulation of the mitochondrial membrane potential [31,32]. Normally, astrocytes express higher steady-state levels of Bcl-2 than neurons [33] suggesting a low vulnerability of astrocytes. Furthermore, in astrocytes oxaliplatin does not increase the expression of the pro-apoptotic protein Bid (able to activate the intrinsic apoptotic process by inducing the cytosolic release of cytochrome C) [34], and Bid basal levels are under the detection threshold. The low basal level of Bid in astrocytes agrees with Harrison et al. [33]. 
Under physiological conditions, glial cells exert neuroprotective effects by providing neurons with substrates for oxidation, regulating the levels of neurotoxic molecules like glutamate [35] and free radicals [36,37]. The induction of apoptosis, the dysregulation of superoxide anion homeostasis [9], and the reduction of Bcl-2 levels in astrocytes suggest that the neurotoxic effects of oxaliplatin derive, at least in part, from an impairment of glial neuroprotective activity.

Caspase- 8 is one of the principal initiator of the extrinsic process and directly activates the effector caspase-3 [38]. The two major pathways to apoptosis are largely independent, although in certain cell types (e.g., hepatocytes) may intersect. Indeed, caspase- 8 can process the pro-apoptotic Bid into its active truncated form transferring the apoptotic information to the mitochondrial process [39]. On the other hand, to prevent catastrophic unscheduled cell death, both pathways are tightly regulated, at multiple steps. Present data show that the apoptotic process activated by oxaliplatin in astrocytes is completely independent from this apoptotic mediator, whereas the anticancer agent significantly increases caspase- 8 activity in the human colon cancer cell line HT-29 and rat PC12 cells (ruling out the possibility that a species-dependent diversity may influence the results). In HT-29 cells, the expression of Bid is increased after oxaliplatin treatment, confirming the activation of the extrinsic pathway. Moreover, Bid seems unable to cross-activate the intrinsic process since cytochrome $\mathrm{C}$ is not significantly released in the cytosol in HT-29. The block of this pathway may be due to the increased levels of Bcl-2 able to protect mitochondria functionality. Bcl-2 is considered a potent multidrug resistance factor [40].

Although ROS can play a role in oxaliplatin-induced apoptosis of human renal cancer cells [41], $\mathrm{O}_{2}{ }^{-}$production is not modified by oxaliplatin in HT-29. These human adenocarcinoma cell line shows a basal $\mathrm{O}_{2}{ }^{-}$production higher than astrocytes, suggesting that these tumoral cells are less susceptible to the oxidative toxic effect and can therefore survive in the presence of high ROS concentrations.

The faster catabolism of cancer cells is lesser sustained by mitochondria in comparison to normal non-neoplastic cells [42]. Tumor cells may produce energy by a high rate of glycolysis followed by lactic acid fermentation in the cytosol. On the contrary, normal cells show a comparatively low rate of glycolysis followed by oxidation of pyruvate in mitochondria. Since glycolysis provides most of the building blocks required for cell proliferation, cancer cells need to activate glycolysis [43]. A lesser sensitivity of the mitochondria functionality against oxaliplatin toxicity in HT-29 in comparison to astrocytes is suggested.

\section{Experimental Section}

\subsection{Cell Cultures}

The human colon cancer cell lines HT-29 and the rat adrenal pheochromocytoma cell line PC12 were obtained from American Type Culture Collection (Rockville, MD, USA). HT-29 were cultured in DMEM high glucose (Invitrogen, Milan, Italy, 11965-092) and PC12 in RMPI 1640 (Invitrogen, 11875-085). Medium for HT-29 was supplemented with 10\% FBS, $2 \mathrm{mM}$ L-glutamine, $100 \mathrm{IU} \cdot \mathrm{mL}^{-1}$ penicillin and $100 \mu \mathrm{g} \cdot \mathrm{mL}^{-1}$ streptomycin (Sigma-Aldrich, Milan, Italy, S6501). Medium for PC12 cells was supplemented with 5\% FBS, $10 \%$ horse serum, $100 \mathrm{IU} \cdot \mathrm{mL}^{-1}$ penicillin and $100 \mu \mathrm{g} \cdot \mathrm{mL}^{-1}$ streptomycin (Sigma-Aldrich, S6501). Cells were maintained at $37{ }^{\circ} \mathrm{C}$ and $5 \% \mathrm{CO}_{2}$ atmosphere. 
Primary cultures of astrocytes were obtained according to the method described by McCarthy and de Vellis [44]. Briefly, the cerebral cortex of newborn (P1-P3) Sprague-Dawley rats (Harlan, Padova, Italy) were dissociated in Hanks' balanced salt solution containing $0.5 \%$ trypsin/EDTA and $1 \%$ DNase (Sigma-Aldrich, T4174 and D4527) for $30 \mathrm{~min}$ at $37{ }^{\circ} \mathrm{C}$. The suspension was mechanically homogenized and filtered. Cells were plated in high-glucose DMEM with 10\% FBS. Confluent primary glial cultures were used to isolate astrocytes, removing microglia and oligodendrocytes by shaking. Immunocytochemistry analysis with GFAP staining revealed the purity of astrocyte cultures. Cells were fixed in 4\% paraformaldehyde, then incubated with the antibody (Dako, Glostrup, Denmark, Z033429, 1:500), and visualized using Alexa Fluor-conjugated secondary antibody (Invitrogen, Milan, Italy, \#A11034, 1:500). Nuclei were stained with 4,6-diamidino-2-phenylindole dihydrochloride. GFAP-positive cells were $90 \%$ in astrocyte cultures. Astrocytes were plated, according to the experimental needs, 21 days after cell isolation. Formal approval to conduct the experiments described was obtained from the Animal Subjects Review Board of the University of Florence. The ethics policy of the University of Florence complies with the Guide for the Care and Use of Laboratory Animals of the U.S. National Institutes of Health (NIH Publication No. 85-23, revised 1996; University of Florence Assurance No. A5278-01).

HT-29, PC12 and astrocyte cells were starved in serum-free DMEM overnight before all treatments. Protein concentrations were measured by bicinchoninc acid assay (Sigma-Aldrich, for Bicinchoninic Acid Solution B9643, for Copper sulphate solution C2284).

Oxaliplatin (Sequoia Research Products, Pangbourne, UK), concentrations and times of incubation were chosen on the basis of previously performed evaluations [9].

\subsection{Cytochrome C Cytosolic Release}

Astrocytes or HT-29 cells were plated in D-polylisinated slides $\left(10^{5}\right.$ cells/slide $)$ and $48 \mathrm{~h}$ after were incubated with $100 \mu \mathrm{M}$ oxaliplatin for $8 \mathrm{~h}$. After treatment, the cells were fixed with $4 \%$ paraformaldehyde in PBS, permeabilized for 10 min with PBS containing 0.1\% Triton X-100, blocked with PBS containing albumin 1\% (Sigma-Aldrich, A2153) and incubated overnight with a rabbit anti-cytochrome $\mathrm{C}$ antibody at $4{ }^{\circ} \mathrm{C}$ (Santa Cruz Biotechnology, Dallas, TX, USA, sc-7159, 1:300). Slides were washed three times with PBS and incubated with the appropriate secondary antibody labeled with Alexa Fluor 488 (Invitrogen, \#A11034, 1:500) at room temperature for $1 \mathrm{~h}$. Images were acquired by a motorized Leica DM6000B microscope equipped with a DFC350FX camera (Leica, Lawrenceville, GA, USA). Cytochrome $\mathrm{C}$ cytosolic levels were assessed by inspection of at least three fields (40X 0.75NA objective) per slide; two slides for each condition were analyzed. Cells displaying a diffuse cytosolic staining pattern were counted using the "cell counter" plugin of ImageJ (1.33 free-share image analysis software, ImageJ, NIH, Bethesda, MD, USA). Results were expressed as percentage of cells displaying a diffuse (released) distribution of cytochrome $C$ [45,46].

\subsection{Superoxide Dismutase (SOD)-Inhibitable Superoxide Anion $\left(\mathrm{O}_{2^{-}}^{-}\right)$Levels}

Astrocytes and HT-29 cells were plated in six-well plates $\left(5 \times 10^{5}\right.$ cells/well for astrocytes and $3 \times 10^{5}$ cells/well for HT-29) and, $48 \mathrm{~h}$ after, they were incubated with or without $100 \mu \mathrm{M}$ oxaliplatin in serum-free DMEM containing cytochrome C from bovine heart (Sigma-Aldrich, C2037, $1 \mathrm{mg} / \mathrm{mL}$ ) 
for $4 \mathrm{~h}$ at $37{ }^{\circ} \mathrm{C}$. Non-specific cytochrome $\mathrm{C}$ reduction was evaluated by carrying out tests in the presence of bovine SOD (Sigma-Aldrich, S9697, $300 \mathrm{mU} / \mathrm{mL}$ ). The supernatants were collected, and the optical density was spectrophotometrically measured at $550 \mathrm{~nm}$. After subtracting the non-specific absorbance, the SOD-inhibitable $\mathrm{O}_{2}{ }^{-}$amount was calculated by using an extinction coefficient of $2.1 \times 10^{4} \mathrm{M}^{-1} \cdot \mathrm{cm}^{-1}$ and expressed as $\mu \mathrm{M} / \mathrm{mg}$ proteins $/ 4 \mathrm{~h}$. The $4 \mathrm{~h}$ incubation interval was chosen on the basis of preliminary experiments, which showed poor reliability for longer cytochrome $\mathrm{C}$ exposure to the cellular environment.

\subsection{Western Blot Analysis}

Astrocytes or HT-29 cells were plated in $25 \mathrm{~cm}^{2}$ cell culture flasks ( $10^{6}$ cells/flask), grown for $48 \mathrm{~h}$ and incubated with $100 \mu \mathrm{M}$ oxaliplatin for $8 \mathrm{~h}$. After treatment, the cells were homogenized in lysis buffer containing $50 \mathrm{mM}$ Tris- $\mathrm{HCl} \mathrm{pH} 8.0,150 \mathrm{mM} \mathrm{NaCl}, 1 \mathrm{mM}$ EDTA, 0.5\% Triton X-100, Complete Protease Inhibitor (Roche, Milan, Italy, 04693124001), and the homogenate was incubated on ice for $30 \mathrm{~min}$. The suspension was sonicated on ice for $15 \mathrm{~s}$. After centrifugation $(13,000 \times g$ for 15 min at $4{ }^{\circ} \mathrm{C}$ ), aliquots containing $35 \mu \mathrm{g}$ total proteins were separated on a $4 \%-12 \%$ sodium dodecyl sulfate (SDS)-polyacrylamide gel by electrophoresis and transferred onto nitrocellulose membranes. Membranes were blocked with 5\% nonfat dry milk in PBS containing 0.1\% Tween 20 (PBST) and then probed overnight with specific primary antibodies. Goat anti-DR5 receptor antibody (Santa Cruz Biotechnology, sc-19529), rabbit anti Bcl-2 antibody (Cell Signaling, Milan, Italy, \#2876) and rabbit anti Bid antibody (Santa Cruz Biotechnology, sc-11423) were diluted to 1:500, 1:1000 and 1:1000, respectively, in 5\% nonfat dry milk in PBST. Membranes were washed with PBST and incubated for $1 \mathrm{~h}$ in PBST containing the appropriate horseradish peroxidase-conjugated secondary antibody (anti rabbit Cell Signaling, \#7074, 1:5000; anti goat Santa Cruz Biotechnology, sc-2020, 1:5000). ECL (Enhanced chemiluminescence; Pierce, Milan, Italy, 32106) was used to visualize the peroxidase-coated bands. Densitometric analysis was performed using the "ImageJ" analysis software (ImageJ, NIH) and results were normalized to GAPDH (Cell signaling, \#2118, 1:2000) immunoreactivity as internal control. Values were reported as percentages in comparison to control, which was arbitrarily fixed at $100 \%$.

\subsection{Caspase-8 Activity}

Astrocytes, HT-29 and PC12 cells were plated in six-well plates $\left(5 \times 10^{5}\right.$ cells/well for astrocytes and $3 \times 10^{5}$ cells/well for HT-29 and PC12) and, after $48 \mathrm{~h}$, they were incubated with $100 \mu \mathrm{M}$ oxaliplatin for $8 \mathrm{~h}$. After treatment, cells were scraped with $70 \mu \mathrm{L}$ of lysis buffer as suggested by the manufacturer (Molecular Probes, Milan, Italy). Fifty microliters of the supernatant were incubated with $50 \mu \mathrm{M}$ of the specific fluorogenic peptide caspase- 8 substrate (IETD-AFC composed by 7-amino-4-trifluoromethyl coumarin and a synthetic tetrapeptide Ile-Glu-Thr-Asp) at $25^{\circ} \mathrm{C}$ for $30 \mathrm{~min}$. The amount of cleaved substrate in each sample was measured in a 96 well plate fluorescence spectrometer (Flexi Station III, Molecular Devices, Sunnyvale, CA, USA; excitation at $400 \mathrm{~nm}$ and emission at $505 \mathrm{~nm})$. 


\subsection{Statistical Analysis}

Results were expressed as mean \pm SEM and analysis of variance (ANOVA) was performed. A Bonferroni's significant difference procedure was used as post hoc comparison. All assessments were made by researchers blinded to cell treatments. Data were analyzed using the "Origin 8.1" software (OriginLab, Northampton, MA, USA).

\section{Conclusions}

Taken together the present data underline that oxaliplatin-dependent apoptosis is mediated preferentially by the intrinsic apoptosis pathway in nervous normal cells. The extrinsic pathway based on caspase- 8 activation strongly participates to apoptosis phenomena in tumor cells. New neuroprotective agents able to preserve mitochondrial functionality without interfere with caspase- 8 activation are suggested as safe candidate to treat oxaliplatin-dependent neurotoxicity.

\section{Supplementary Materials}

Supplementary materials can be found at http://www.mdpi.com/1422-0067/16/03/5386/s1.

\section{Acknowledgments}

This research was funded by the Italian Ministry of Instruction, University and Research (MIUR) and by the University of Florence.

\section{Author Contributions}

Lorenzo Di Cesare Mannelli, Paola Failli and Carla Ghelardini conceived and designed the experiments; Matteo Zanardelli and Laura Micheli performed the experiments; Matteo Zanardelli analyzed the data; Lorenzo Di Cesare Mannelli, Paola Failli and Matteo Zanardelli wrote the paper; Raffaella Nicolai participated in the design of the study and helped to draft the manuscript.

\section{Conflicts of Interest}

The authors declare no conflict of interest.

\section{References}

1. André, T.; Boni, C.; Mounedji-Boudiaf, L.; Navarro, M.; Tabernero, J.; Hickish, T.; Topham, C.; Zaninelli M.; Clingan, P.; Bridgewater, J.; et al. Oxaliplatin, fluorouracil, and leucovorin as adjuvant treatment for colon cancer. N. Engl. J. Med. 2004, 350, 2343-2351.

2. Hoff, P.M.; Saad, E.D.; Costa, F.; Coutinho, A.K.; Caponero, R.; Prolla, G.; Gansl, R.C. Literature review and practical aspects on the management of oxaliplatin-associated toxicity. Clin. Colorectal Cancer 2012, 11, 93-100.

3. Kidani, Y.; Noji, M.; Tashiro, T. Antitumor activity of platinum (II) complexes of 1,2-diamino-cyclohexane isomers. Gann 1980, 71, 637-643. 
4. Tashiro, T.; Kawada, Y.; Sakurai, Y.; Kidani, Y. Antitumor activity of a new platinum complex, oxalato (trans-1-1,2-diaminocyclohexane) platinum (II): New experimental data. Biomed. Pharmacother. 1989, 43, 251-260.

5. De Gramont, A.; Figureer, A.; Seymour, M.; Homerin, M.; Hmissi, A.; Cassidy, J.; Boni, C.; Cortes-Funes, H.; Cervantes, A.; Freyer, G.; et al. Leucovorin and fluorouracil with or without oxaliplatin as first-line treatment in advanced colorectal cancer. J. Clin. Oncol. 2000, 18, 2938-2947.

6. Krishnan, A.V.; Goldstein, D.; Friedlander, M.; Kiernan, M.C. Oxaliplatin-induced neurotoxicity and the development of neuropathy. Muscle Nerve 2005, 32, 51-60.

7. Di Cesare Mannelli, L.; Zanardelli, M.; Failli, P.; Ghelardini, C. Oxaliplatin-induced neuropathy: Oxidative stress as pathological mechanism. Protective effect of silibinin. J. Pain 2012, 13, 276-284.

8. Zanardelli, M.; Micheli, L.; Cinci, L.; Failli, P.; Ghelardini, C.; di Cesare Mannelli, L. Oxaliplatin neurotoxicity involves peroxisome alterations. PPAR $\gamma$ agonism as preventive pharmacological approach. PLoS One 2014, 9, e102758.

9. Di Cesare Mannelli, L.; Zanardelli, M.; Failli, P.; Ghelardini, C. Oxaliplatin-induced oxidative stress in nervous system-derived cellular models: Could it correlate with in vivo neuropathy? Free Radic. Biol. Med. 2013, 61, 143-150.

10. Galluzzi, L.; Blomgren, K.; Kroemer, G. Mitochondrial membrane permeabilization in neuronal injury. Nat. Rev. Neurosci. 2009, 10, 481-494.

11. Khan, K.H.; Blanco-Codesido, M.; Molife, L.R. Cancer therapeutics: Targeting the apoptotic pathway. Crit. Rev. Oncol. Hematol. 2014, 90, 200-219.

12. Forbes-Hernández, T.Y.; Giampieri, F.; Gasparrini, M.; Mazzoni, L.; Quiles, J.L.; Alvarez-Suarez, J.M.; Battino, M. The effects of bioactive compounds from plant foods on mitochondrial function: A focus on apoptotic mechanisms. Food Chem. Toxicol. 2014, 68, 154-182.

13. Hsu, Y.T.; Youle, R.J. Bax in murine thymus is a soluble monomeric protein that displays differential detergent-induced conformations. J. Biol. Chem. 1998, 273, 10777-10783.

14. Lalier, L.; Cartron, P.F.; Juin, P.S.; Nedelkina, S.; Manon, S.; Bechinger, B.; Vallette, F.M. Bax activation and mitochondrial insertion during apoptosis. Apoptosis 2007, 12, 887-896.

15. Kang, M.H.; Reynolds, C.P. Bcl-2 inhibitors: Targeting mitochondrial apoptotic pathways in cancer therapy. Clin. Cancer Res. 2009, 15, 1126-1132.

16. Petros, A.M.; Olejniczak, E.T.; Fesik, S.W. Structural biology of the Bcl-2 family of proteins. Biochim. Biophys. Acta 2004, 1644, 83-94.

17. Ashkenazi, A.; Dixit, V.M. Death receptors: Signaling and modulation. Science 1998, 281, 1305-1308.

18. Ashkenazi, A. Targeting death and decoy receptors of the tumour-necrosis factor superfamily. Nat. Rev. Cancer 2002, 2, 420-430.

19. Yin, X.M. Signal transduction mediated by Bid, a pro-death Bcl-2 family proteins, connects the death receptor and mitochondria apoptosis pathways. Cell Res. 2000, 10, 161-167.

20. Ashkenazi, A. Directing cancer cells to self-destruct with pro-apoptotic receptor agonists. Nat. Rev. Drug Discov. 2008, 7, 1001-1012. 
21. Kluck, R.M.; Bossy-Wetzel, E.; Green, D.R.; Newmeyer, D.D. The release of cytochrome c from mitochondria: A primary site for bcl-2 regulation of apoptosis. Science 1997, 275, 1132-1136.

22. Lim, M.L.; Lum, M.G.; Hansen, T.M.; Roucou, X.; Nagley, P. On the release of cytochrome c from mitochondria during cell death signaling. J. Biomed. Sci. 2002, 9, 488-506.

23. Zheng, H.; Xiao, W.H.; Bennett, G.J. Functional deficits in peripheral nerve mitochondria in rats with paclitaxel- and oxaliplatin-evoked painful peripheral neuropathy. Exp. Neurol. 2011, 232, $154-161$.

24. Marchi, S.; Giorgi, C.; Suski, J.M.; Agnoletto, C.; Bononi, A.; Bonora, M.; de Marchi, E.; Missiroli, S.; Patergnani, S.; Poletti, F.; et al. Mitochondria-ros crosstalk in the control of cell death and aging. J. Signal Transduct. 2012, 2012, doi:10.1155/2012/329635.

25. Emerit, J.; Edeas, M.; Bricaire, F. Neurodegenerative diseases and oxidative stress. Biomed. Pharmacother. 2004, 58, 39-46.

26. Almeida, A.; Delgado-Esteban, M.; Bolanos, J.P.; Medina, J.M. Oxygen and glucose deprivation induces mitochondrial dysfunction and oxidative stress in neurons but not in astrocytes in primary culture. J. Neurochem. 2002, 81, 207-217.

27. Han, D.; Williams, E.; Cadenas, E. Mitochondrial respiratory chain-dependent generation of superoxide anion and its release into the intermembrane space. Biochem. J. 2001, 353, 411-416.

28. Muller, F.L.; Liu, Y.; van Remmen, H. Complex III releases superoxide to both sides of the inner mitochondrial membrane. J. Biol. Chem. 2004, 279, 49064-49073.

29. Mayer, B.; Oberbauer, R. Mitochondrial regulation of apoptosis. News Physiol. Sci. 2003, 18, 89-94.

30. Gottlieb, R.A. Mitochondria and apoptosis. Biol. Signals Recept. 2001, 10, 147-161.

31. Polster, B.M.; Kinnally, K.W.; Fiskum, G. BH3 death domain peptide induces cell type-selective mitochondrial outer membrane permeability. J. Biol. Chem. 2001, 276, 37887-37894.

32. Shimizu, S.; Tsujimoto, Y. Proapoptotic BH3-only Bcl-2 family members induce cytochrome $c$ release, but not mitochondrial membrane potential loss, and do not directly modulate voltage-dependent anion channel activity. Proc. Natl. Acad. Sci. USA 2000, 97, 577-582.

33. Harrison, J.F.; Rinne, M.L.; Kelley, M.R.; Druzhyna, N.M.; Wilson, G.L.; Ledoux, S.P. Altering DNA base excision repair: Use of nuclear and mitochondrial-targeted $N$-methylpurine DNA glycosylase tosensitize astroglia to chemotherapeutic agents. Glia 2007, 55, 1416-1425.

34. Cory, S.; Adams, J.M. The Bcl2 family: Regulators of the cellular life-or-death switch. Nat. Rev. Cancer 2002, 2, 647-656.

35. Hansson, E.; Rönnbäck, L. Astrocytes in glutamate neurotransmission. FASEB J. 1995, 9, 343-350.

36. Shih, A.Y.; Johnson, D.A.; Wong, G.; Kraft, A.D.; Jiang, L.; Erb, H.; Johnson, J.A.; Murphy, T.H. Coordinate regulation of glutathione biosynthesis and release by Nrf2-expressing glia potently protects neurons from oxidative stress. J. Neurosci. 2003, 23, 3394-3406.

37. Kraft, A.D.; Johnson, D.A.; Johnson, J.A. Nuclear factor E2-related factor 2-dependent antioxidant response element activation by tert-butylhydroquinone and sulforaphane occurring preferentially in astrocytes conditions neurons against oxidative insult. J. Neurosci. 2004, 24, 1101-1112. 
38. Ashkenazi, A.; Holland, P.; Eckhardt, S.G. Ligand-based targeting of apoptosis in cancer: The potential of recombinant human apoptosis ligand 2/Tumornecrosis factor-related apoptosis-inducing ligand (rhApo2L/TRAIL). J. Clin. Oncol. 2008, 26, 3621-3630.

39. Adams, J.M.; Cory, S. The Bcl-2 apoptotic switch in cancer development and therapy. Oncogene 2007, 26, 1324-1337.

40. Lacour, S.; Micheau, O.; Hammann, A.; Drouineaud, V.; Tschopp, J.; Solary, E.; Dimanche-Boitrel, M.T. Chemotherapy enhances TNF-related apoptosis-inducing ligand DISC assembly in HT-29 human colon cancer cells. Oncogene 2003, 22, 1807-1816.

41. Kim, S.; Lee, T.J.; Park, J.W.; Kwon, T.K. Overexpression of cFLIPs inhibits oxaliplatin-mediated apoptosis through enhanced XIAP stability and Akt activation in human renal cancer cells. J. Cell Biochem. 2008, 105, 971-979.

42. Kim, J.W.; Dang, C.V. Cancer's molecular sweet tooth and the Warburg effect. Cancer Res. 2006, 66, 8927-8930.

43. Lopez-Lazaro, M. The Warburg effect: Why and how do cancer cells activate glycolysis in the presence of oxygen? Anticancer Agents Med. Chem. 2008, 8, 305-312.

44. McCarty, K.D.; de Vellis, J. Preparation of separate astroglial and oligodendroglial cell cultures from rat cerebral tissue. J. Cell Biol. 1980, 85, 890-902.

45. Waterhouse, N.J.; Trapani, J.A. A new quantitative assay for cytochrome c release in apoptotic cells. Cell Death Differ. 2003, 10, 853-855.

46. Goldstein, J.C.; Muñoz-Pinedo, C.; Ricci, J.E.; Adams, S.R.; Kelekar, A.; Schuler, M.; Tsien, R.Y.; Green, D.R. Cytochrome c is released in a single step during apoptosis. Cell Death Differ. 2005, $12,453-462$.

(C) 2015 by the authors; licensee MDPI, Basel, Switzerland. This article is an open access article distributed under the terms and conditions of the Creative Commons Attribution license (http://creativecommons.org/licenses/by/4.0/). 